\title{
An Elastic Lattice in a Random Potential
}

\author{
Eugene M. Chudnovsky and Ronald Dickman ${ }^{\dagger}$ \\ Department of Physics and Astronomy, Lehman College, CUNY, Bedford Park Boulevard West, \\ Bronx, NY 10468-1589
}

(September 26, 2018)

\begin{abstract}
Using Monte Carlo simulations, we study the properties of an elastic triangular lattice subject to a random background potential. As the cooling rate is reduced, we observe a rather sudden crossover between two different glass phases, one with exponential decay of translational correlations, the other with power-law decay. Contrary to predictions derived from continuum models, no evidence of a crossover in the mean-square displacement, $B(r)$, from quadratic growth at small $r$, to logarithmic growth at large $r$ is found.
\end{abstract}

PACS numbers: 74.60.Ge, 05.20.-y

${ }^{\dagger}$ e-mail address: dickman@lcvax.lehman.cuny.edu 
The structure of an elastic lattice in a random background has received much attention in recent years, due to its relevance to such systems as a Wigner crystal in a semiconductor with impurities [1], a charge density wave in a weakly disordered medium [2], an atomic monolayer on an imperfect crystal surface [3], a magnetic bubble lattice in a ferromagnetic film with defects [ [4], and a vortex lattice in a disordered superconductor [5]. The latter problem has been investigated most intensively, both theoretically and experimentally, because translational correlations in the vortex lattice are responsible for such important properties of superconductors as resistivity and critical current.

Most theoretical works on elastic lattices in a random background have concentrated on a continuum approach in which the deformation of the lattice is described by the displacement field $\mathbf{u}(\mathbf{r})$. In this model the background is described by a random potential $V[\mathbf{r}, \mathbf{u}(\mathbf{r})]$ that satisfies $V\left[\mathbf{r}, \mathbf{u}(\mathbf{r})+\mathbf{a}_{i}\right]=V[\mathbf{r}, \mathbf{u}(\mathbf{r})]$ which accounts for the periodicity of the lattice. ( $\mathbf{a}_{i}$ is a lattice vector.) The energy of this system is

$$
U=\int d^{d} r[\hat{\alpha} \nabla \mathbf{u} \nabla \mathbf{u}+V(\mathbf{r}, \mathbf{u})],
$$

where the first term represents elasticity; $\alpha_{i k l m}$ being the tensor of elastic moduli. When $V=$ 0 , that is in the absence of pinning, the energy is minimized by $\mathbf{u}=$ const. which represents perfect translational order. In the presence of pinning the lattice develops deformations, $\mathbf{u}(\mathbf{r})$, and the question arises whether (and how rapidly) these deformations destroy long range translational correlations. A convenient measure of the disorder is

$$
B(\mathbf{r})=<[\mathbf{u}(\mathbf{r})-\mathbf{u}(0)]^{2}>
$$

If pinning is weak and only a small area of the lattice is of interest, the deformation $u$ is small compared to the lattice spacing $a$. Then $V(\mathbf{r}, \mathbf{u})$ can be written as $-\mathbf{f}(\mathbf{r}) \cdot \mathbf{u}$ and the problem reduces to the random force problem, for which the solution is known: a random force, no matter how weak, destroys the long range translational order in less than four dimensions. Simple statistical arguments yield [6:0] $B \sim\left(f^{2} / \alpha^{2}\right) r^{4-d}$, where $f^{2}$ denotes the variance of the random force. That is, $B \propto r$ in three dimensions and $B \propto r^{2}$ in two dimensions. Equating $B$ to $a$ yields an estimate of the translational correlation length, $\xi \propto f^{2 /(d-4)}$. This estimate seems to be quite robust with respect to approximations and assumptions about the random potential. It has been pointed out, however, that the random field model cannot provide the correct rate at which translational correlations are destroyed for $r>\xi$, as it does not take into account the periodicity of the lattice. More sophisticated approaches based upon the Gaussian variational method [8] and the functional renormalization group [9] suggest that $B \propto(4-d) \ln r$ for $r>\xi$ [10 12]. Another renormalization group approach [13] suggests that the disordered lattice freezes below a certain temperature into a glass state with $B \propto \ln ^{2} r$. The upshot of these analytical results is a much slower decay of translational correlations than predicted by the random field model [6, ].

The purpose of this paper is to compare the above predictions of the continuous model with Monte Carlo simulations of a discrete triangular lattice subject to a random background potential. As in the continuous approaches mentioned above, we limit our consideration to lattices free of dislocations. The results obtained under this assumption may be relevant to real systems since patterns of vortex lattices observed in decoration experiments show remarkably large areas free of dislocations. 
We consider a two-dimensional triangular lattice of particles coupled by a harmonic, nearest-neighbor interaction, and subject to a static, random potential $V(x, y)$. Let $\mathbf{x}_{i}=$ $\left(x_{i}, y_{i}\right)$ denote the position of particle $i$. Then the potential energy of the system is

$$
E=\frac{1}{2} \sum_{<i, j>}\left(r_{i, j}-1\right)^{2}+\sum_{i} V\left(\mathbf{x}_{i}\right), \quad,
$$

where the first sum is over all nearest-neighbor pairs in the triangular lattice, and $r_{i, j}=$ $\left|\mathbf{x}_{\mathbf{i}}-\mathbf{x}_{\mathbf{j}}\right|$. (All quantities are dimensionless in our formulation, with the basic length scale $a=1$ set by the nearest-neighbor $(\mathrm{NN})$ separation in the unstrained lattice and the basic energy scale $e=1 / 2$ set by the energy of a NN pair with $|r-1|=1$, so that the spring constants have unit magnitude.)

To avoid severe distortions of the lattice we impose a planarity constraint, which prevents any particle from escaping the "cage" defined by the current positions of its six nearest neighbors. (This is enforced by demanding that if $\mathbf{x}, \mathbf{y}$ and $\mathbf{z}$ form a unit triangle in the unstrained lattice, then the angle between $\mathbf{x}-\mathbf{y}$ and $\mathbf{z}-\mathbf{y}$ not exceed $\pi$.) We adopted this constraint in preference to equipping the particles with hard cores, since hard cores of a size sufficient to prevent this kind of distortion yield a rather strong anharmonicity. In the present model the anharmonicity is weak, with the deviation from equipartition amounting to $<5 \%$ at the temperatures of interest.

The background potential $V(x, y)$ is generated in two steps. We first generate $R(i, j)$, an $N \times N$ array (we used $N=101$ ) of uncorrelated random numbers, uniformly distributed on $[-1 / 2,1 / 2]$. We smooth this array by replacing each entry $R(i, j)$ by the sum of the entry and its four nearest-neighbors, using periodic boundaries at the edges. The smoothing process is applied a total of three times, so that elements up to six units apart have a nonzero correlation. (Each entry of $R$ is now a weighted sum of 25 random numbers, and so represents a good approximation to a Gaussian random variable.) To find $V(x, y)$, we define a random, piece-wise constant (on a scale of $\sim 10^{-3}$ ) map between points $(x, y)$ and a set of four entries $R_{1}, \ldots, R_{4}$, where $R_{k} \equiv R\left(i_{k}, j_{k}\right)$. The first entry is given by the modular expression: $i_{1}=$ [7x $(\bmod 101)]$, where the brackets denote the largest integer, and similarly for $j_{1}$. Then $i_{2}=$ $\left[f i_{1}(\bmod 100)\right]$, where $f \equiv 2049\left|R_{1}\right|$ is a random multiplier; $j_{2}$ is defined similarly, yielding $R_{2}$. We repeat the process, this time using $f=513\left|R_{1}+R_{2}\right|$, and then once more, with $f=257\left|R_{1}+R_{2}+R_{3}\right|$. The resulting $V(x, y) \equiv A \sum_{i=1}^{4} R_{i}$ is an (approximately) Gaussian random field with short-range correlations; $C_{V}(r) \equiv\langle V(\mathbf{x}) V(\mathbf{y})\rangle_{|\mathbf{x}-\mathbf{y}|=r} / \sigma^{2}$ drops from unity to about 0.3 for $r \simeq 10^{-3}$, and then decays in roughly linear fashion, remaining essentially zero for $r \geq 0.6$. (We found that choosing prime factors in the multipliers hastened the decay of correlations.) The strength of the background potential is characterized by its standard deviation, $\sigma$, which we control by varying the constant $A$. $(\sigma=0.2$ and 0.5 are used in this study. All simulations were performed on DEC Alpha workstations, and employed the random number generator supplied with the machine.)

Thus the background potential differs from the kind typically employed in studies of off-lattice systems subject to quenched randomness which distributes a certain density of identical centers of force, with potential $v(r)$, at random positions, $\mathbf{q}_{i}$, and sets $V(\mathbf{x})=$ $\sum_{i} v\left(\left|\mathbf{x}-\mathbf{q}_{i}\right|\right)$. Our potential is a closer approximation to the Gaussian random field used in theoretical analyses.

We simulated hexagonal-shaped lattices of $M$ particles to a side (a total of $3 M(M-1)+1$ 
particles), with open boundaries. We report results for $M=60$ and $M=120$. We used open boundaries to eliminate global periodicity as a restraint on the growth of particle displacements. In each step of the simulation, a particle is selected at random and subjected to a trial displacement uniform on a square of side $D=0.5$, symmetric about the origin. The move is accepted if the total change in energy $\Delta E \leq 0$; if $\Delta E$ is positive the new position is accepted with probability $e^{-\Delta E / T}$. Our time unit comprises one attempted move per particle. A preliminary study of the lattice without the random background potential (see also Ref. [14]) revealed that the correlation function

$$
g_{\mathbf{G}}(\mathbf{r})=\left\langle e^{i \mathbf{G} \cdot\left(\mathbf{x}_{i}-\mathbf{x}_{j}\right)}\right\rangle,
$$

(the thermal average is over all pairs with $\mathbf{x}_{i}-\mathbf{x}_{j}=\mathbf{r}$ in the unstrained lattice; $\mathbf{G}$ is a reciprocal lattice vector), shows a power-law decay, $g_{\mathbf{G}}(\mathbf{r}) \sim r^{-\eta}$ with $\eta$ proportional to temperature, $T$, as expected [15].

Our primary interest is in the behavior of the mean-square displacement from equilibrium,

$$
B(\mathbf{r}) \equiv<\left[\mathbf{x}_{i}-\mathbf{x}_{j}-\mathbf{r}\right]^{2}>,
$$

where we average over all pairs with $\mathbf{x}_{i}-\mathbf{x}_{j}=\mathbf{r}$ in the unstrained lattice, and the angle brackets denote an average over disorder. To avoid the effects of strong distortions that may appear at the boundary, we only average over particles at least $M / 2$ sites distant from it. Configurations are generated by taking the perfect, unstrained lattice and permitting it to relax, in the presence of $V(\mathbf{x})$, for $t_{A}=10^{4}-10^{5}$ timesteps at temperature 1 . After this "annealing" phase the system is gradually cooled: the inverse temperature $\beta=1 / T$ increases at a constant cooling rate $\Gamma$, until $T=0.01$, at which time we compute $B$ and other properties. (At this point the system is essentially at temperature zero, since the typical background energy $\sigma \gg T$.)

Depending on the cooling rate, we observe two qualitatively distinct kinds of $B(r)$. In Fig. 1, for example, we show $B(r)$ for $M=60, \sigma=0.2, t_{A}=10^{4}$, and a variety of different cooling rates. For relatively rapid cooling, $\left(\Gamma>4 \times 10^{-4}\right.$ for the parameters of Fig. 1), $B$ grows $\sim \ln r$ for small $r$ before crossing over to some slower growth whose precise form is unclear. The correlation length $\xi$, defined via $B(\xi)=1$, decreases with $\Gamma$ and in many cases exceeds the system size $M$. The logarithmic growth is reminiscent of a lattice in thermal equilibrium [15,14, suggesting that in this case the system has undergone a kind of glass transition, the final $B(r)$ being a remnant of the thermal disorder when the lattice fell out of equilibrium with the heat bath. In this situation the background potential serves to "freeze" thermal fluctuations, even though the system does not have time to optimize its configuration with respect to $V(\mathbf{r})$. Fig. 2 illustrates the similarity between $B(r)$ in thermal equilibrium and in a rapidly cooled system.

For lower cooling rates, the particles have the opportunity to explore more of the local potential energy landscape, and the lattice distortion, while more modest for small $r$, becomes sizeable on large scales. In this regime the correlation length decreases as we reduce $\Gamma$; increased exploration results in greater distortion. An important open question concerns the nature of $B(r)$ in the ground state: Does it grow $\sim r^{2}$ indefinitely, or cross over to a slower, perhaps logarithmic growth law? As we reduce the cooling rate, and hence probe nearer the ground state of the model, we find that $B(r)$ maintains a faster than linear growth. Fig. 3 
shows that in this regime, $B(\mathbf{r}) \sim r^{2}$ to a good approximation. In no instance do we observe a crossover to logarithmic growth at large $r$, even though our data extend to $r / \xi \simeq 14$ for some systems.

Fig. 4 shows the elastic and potential energies ( $u$ and $v$, respectively), per particle as a function of $\Gamma$, for the conditions of Fig. 1. While there are some fluctuations (due to computer-time restrictions we average over sets of only five independent disorder configurations in this series of studies), the energy decreases with cooling rate, as expected. We also plot $B(r=50)$ as a measure of the character of the final state (quenched thermal fluctuations versus optimized in the random background). It appears that the change in the behavior of $B$ occurs rather suddenly, at $\Gamma \simeq 4 \times 10^{-4}$.

In summary, we studied the mean-square displacement in an elastic lattice subject to a static random potential, and find no evidence of crossover to logarithmic growth in the well-relaxed regime. A crossover between two different glass phases, one with exponential decay of translational correlations, the other having power-law decay, is observed as the cooling rate is reduced.

This work was supported by the Department of Energy under Grant No. DE-FG02$93 \mathrm{ER} 45487$. 


\section{REFERENCES}

[1] E. Y. Andrei et al., Phys. Rev. Lett. 60, 2765 (1988).

[2] H. Dai, H. Chen, and C. M. Lieber, Phys. Rev. Lett. 66, 3183 (1991); H. Dai and C. M. Lieber, ibid. 69, 1576 (1992).

[3] S. E. Nagler et al., Phys. Rev. B32, 7373 (1985); N. Greiser et al., Phys. Rev. Lett. 59, 1706 (1987).

[4] R. Seshardi and R. M. Westervelt, Phys. Rev. Lett. 66, 2774 (1991); Phys. Rev. B46, 5142 (1992).

[5] C. A. Murray et al., Phys. Rev. Lett. 64, 2312 (1990); C. A. Bolle et al., ibid. 66, 112 (1991); D. G. Grier et al., ibid. 66, 2270 (1990).

[6] A I. Larkin, Zh. Eksp. Teor. Fiz. 58, 1466 (1970) [Sov. Phys.JETP 31, 784 (1970)]; A. I. Larkin and Yu.M. Ovchinnikov, J. Low Temp. Phys. 34, 409 (1979); E. H. Brandt, J. Low Temp. Phys. 64, 375 (1986); M. V. Feigel'man, V. B. Geshkenbein, A. I. Larkin, and V. M. Vinokur, Phys. Rev. Lett. 63, 2303 (1989).

[7] Y. Imry and S. Ma, Phys. Rev. Lett. 35, 1399 (1975).

[8] M. Mezard and G. Parisi, J. Phys. I (Paris) 4, 809 (1991).

[9] D. Fisher, Phys. Rev. Lett. 56, 1964 (1986).

[10] T. Nattermann, Phys. Rev. Lett. 64, 2454 (1990).

[11] T. Giamarchi and P. Le Doussal, Phys. Rev. Lett. 72, 1530 (1994).

[12] J.-P. Bouchaud, M. Mezard, and J. S. Yedidia, Phys. Rev. Lett. 67, 3840 (1991).

[13] C. Carraro and D. R. Nelson, preprint.

[14] R. Dickman and E. M. Chudnovsky, Phys. Rev. B51, 97 (1995).

[15] B. I. Halperin and D. R. Nelson, Phys. Rev. Lett. 41, 121 (1978); D. R. Nelson and B. I. Halperin, Phys.Rev. B19, 2457 (1979). 


\section{Figure Captions}

FIG. 1. $B(r)$ versus $r$ for $M=60, \sigma=0.2, t_{A}=10^{4}$, and various cooling rates $\Gamma$.

FIG. 2. $B(r)$ versus $\ln r$ for $M=60$ and $\sigma=0.2$. Solid line: system in equilibrium at $T=0.3$; broken line: $T=0.2$; circles: system cooled to $T=0.01$ at $\Gamma=1$; squares: $\Gamma=0.5$.

FIG. 3. Mean-square displacement $B(r)$ versus $\tilde{r} \equiv r / \xi$. Dashed line: $M=120, \sigma=0.5$, $\Gamma=5 \times 10^{-4}$; solid line: $M=120, \sigma=0.2, \Gamma=10^{-3}$; dotted line: $M=60, \sigma=0.2$, $\Gamma=10^{-4}$.

FIG. 4. Per-particle potential energy, $v$, elastic energy, $u$, and $B(r=50)$ versus $\Gamma$ for the same parameters as in Fig. 1. 


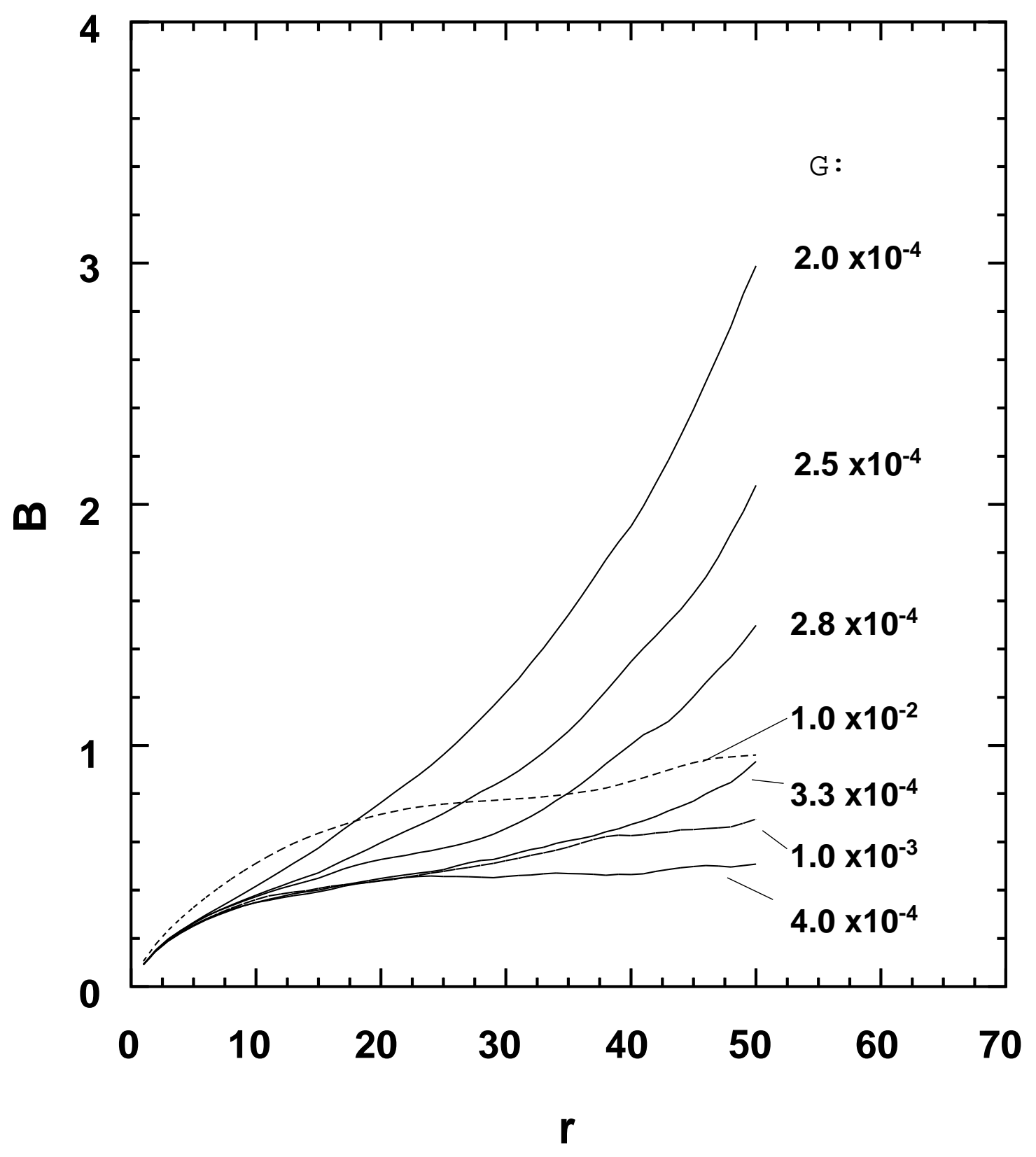

Fig. 1 


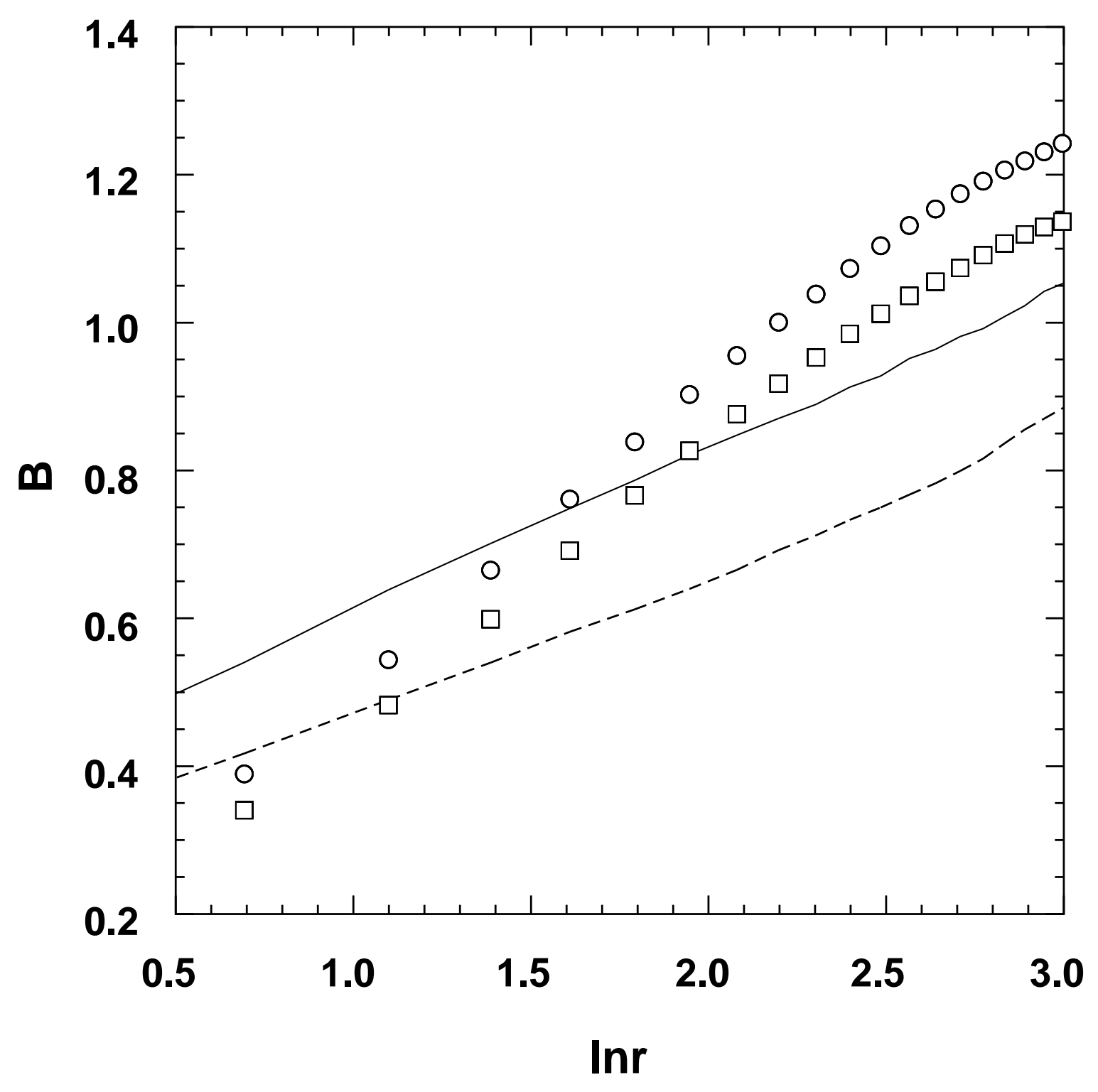

Fig. 2 


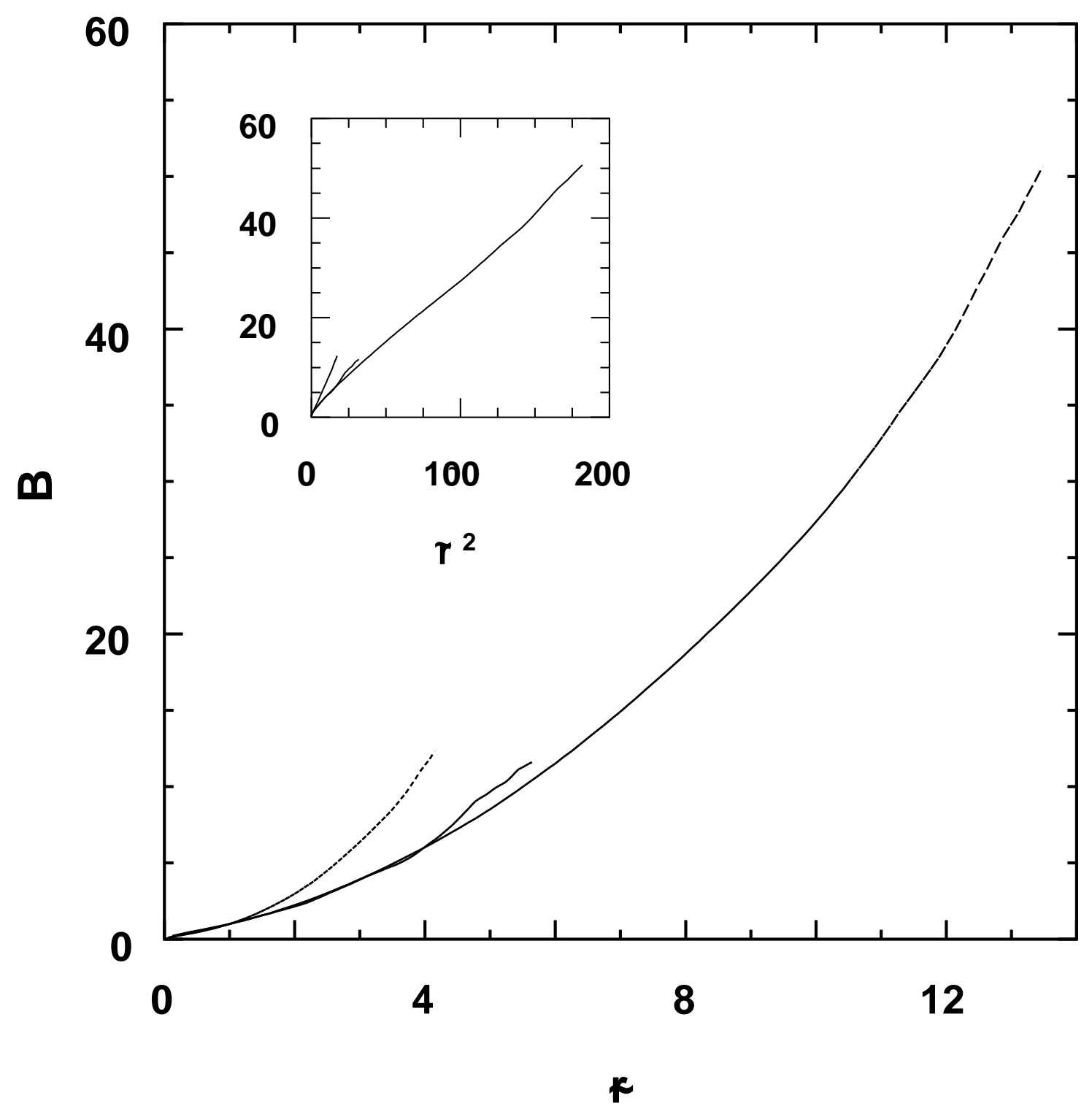

Fig. 3 

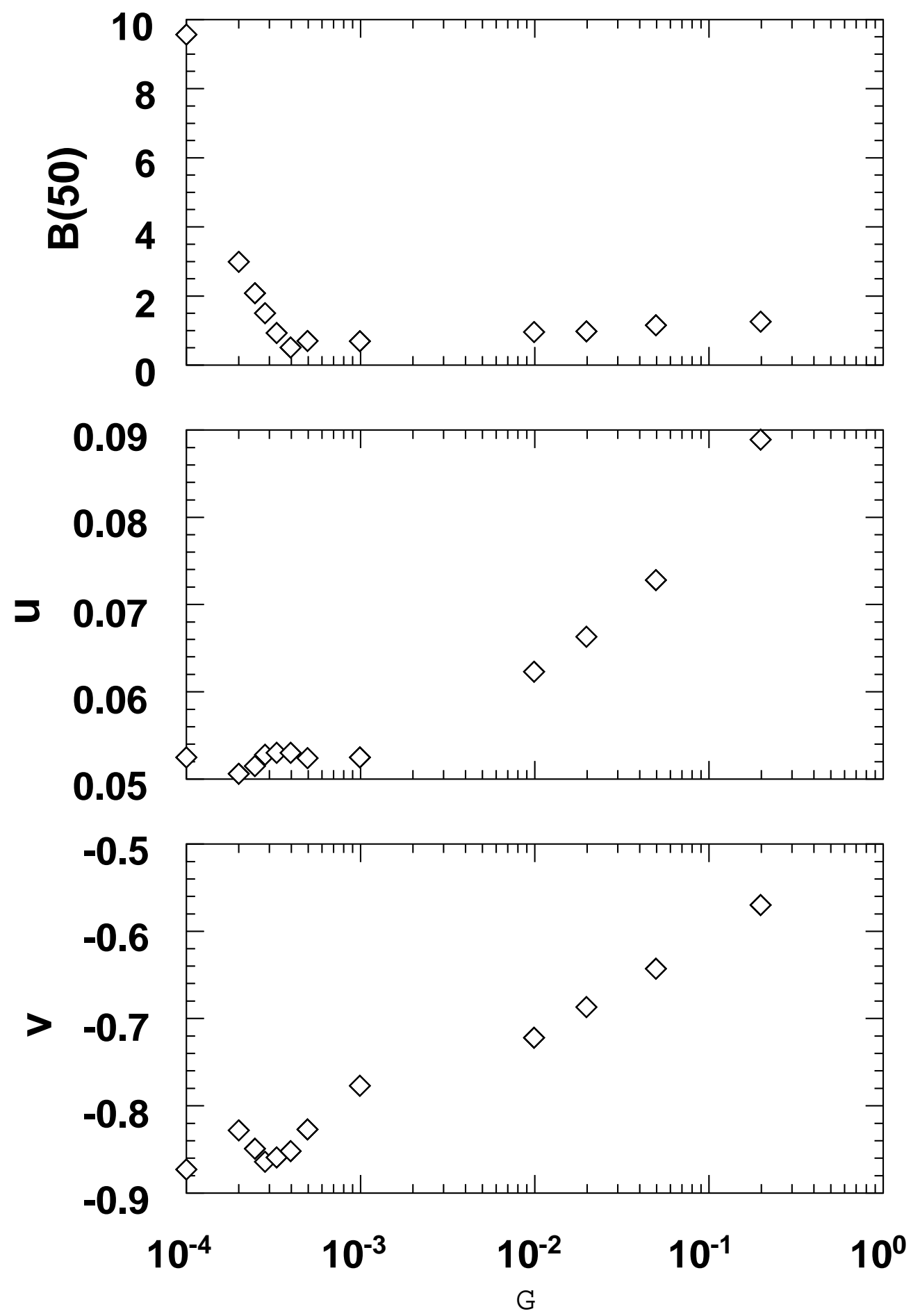

Fig. 4 